\title{
Therapeutic Effect
}

National Cancer Institute

\section{Source}

National Cancer Institute. Therapeutic Effect. NCI Thesaurus. Code C38032.

Beneficial responses or outcomes in the prevention, treatment, or progression of a disease resulting from treatment intervention. Therapeutic effect includes prevention or reduction of adverse effects. ( $\mathrm{NCl}$ ) 